\title{
THE THERMOELASTIC STATE OF A BI-MATERIAL WITH AN OPEN GAS-FILLED INTERFACE CRACK
}

\author{
Khristina Serednytska, Rostyslav Martynyak, Kostyantyn Chumak \\ Pidstryhach Institute for Applied Problems of Mechanics and Mathematics, NASU, Lviv, Ukraine \\ e-mail:labmtd@iapmm.lviv.ua
}

\begin{abstract}
The thermoelastic behavior of a bi-material with a gas-filled interface crack is investigated. The bi-material is subjected to a uniform tensile load and a uniform heat flow. The gas exerts pressure on the crack surfaces and offers thermal resistance proportional to the crack opening. The gas state is assumed to be described by the ideal gas law. The effects of gas mass, gas thermal conductivity and heat flux on the crack opening, interface temperature jump, gas pressure and stress intensity factors are analyzed. It is revealed that a bi-material with a heat-conducting crack exhibits the heat flow directional effect.
\end{abstract}

Keywords: bi-material, interface crack, ideal gas, pressure, thermal resistance

\section{Introduction}

Open internal and interface cracks in natural and technical structures can be filled with a certain medium (liquid and/or gas) that penetrates from an environment, seeps or diffuses from the surrounding material. The crack filler exerts pressure on the crack surfaces and thus affects the distribution of stresses in the vicinity of the crack, and may cause its propagation. The stress-strain state of homogeneous and piecewise-homogeneous bodies with open cracks filled with a liquid or a gas, the pressure of which depends on the applied load, was investigated by Alekseev et al. (1992), Andreikiv and Hembara (2008), Balueva and Goldstein (1995), Evtushenko and Sulim (1981), Feraille-Fresnet et al. (2003), Kaczyński and Monastyrskyy (2004), and Monastyrskyy and Kaczyński (2007).

If bodies are heated, the heat transfer across an open crack and its filler occurs. It affects distributions of temperature and thermal stresses in the vicinity of the crack. To describe the heat transfer across the crack filler, Podstrigach and Kit (1967) applied Podstrigach's model of non-perfect thermal contact through the interstitial layer (Podstrigach, 1963), which was formulated in the form of two equations linking boundary values of temperature and heat flows on the crack surfaces. These equations include two prescribed effective thermal characteristics of a crack - a transverse thermal conductance $\lambda_{n}$ and a longitudinal thermal conductance $\lambda_{s}$. Based on the physical essence of the effective characteristics $\lambda_{n}$ and $\lambda_{s}$, Shvets and Martynyak (1985) expressed them through the gap opening $h(x)$ and the thermal conductivity $\lambda_{c}$ of the gap filler $\left(\lambda_{n}(x)=\lambda_{c} / h(x), \lambda_{s}(x)=\lambda_{c} h(x)\right)$ when investigating thermo-stressed contact of bodies with interface gaps.

Since thermal conductivity of a gas in a crack is much smaller than thermal conductivities of the surrounding materials, and the crack opening is of the order of elastic displacements and is much less than the crack length, the heat transfer across the crack greatly exceeds the heat transfer along the crack. The effective longitudinal thermal conductance $\lambda_{s}$ can therefore be neglected $\left(\lambda_{s}=0\right)$. In this case, the normal heat flow on the crack surfaces is continuous and the temperature jump $\Delta T(x)=r_{n}(x) q_{n}(x)\left(r_{n}(x)=1 / \lambda_{n}(x)\right.$ - thermal resistance of the crack) occurs between the crack surfaces. Martynyak and Chumak (2009) and Chumak and Martynuk 
(2012) used such a formulation to investigate the thermo-stressed contact between bodies with filled interface grooves.

Matczyński et al. (1999) constructed an approximate analytical solution of the plane thermoelastic problem for a homogeneous isotropic body with a crack of variable height, filled with an ideal gas, taking into consideration gas pressure and crack thermal resistance dependent on the crack opening. Two cases were investigated: a) a completely open crack for the initial range of compressive load; b) partial contact of the crack surfaces near the crack tips for the load exceeding some critical load.

Applying the partially-thermally permeable crack model, Zhong and Lee (2012) and Zhong and $\mathrm{Wu}$ (2012) solved the plane thermoelastic contact problems for a transversely isotropic material and an orthotropic material with an open crack filled with a heat-conducting medium. $\mathrm{Li}$ and Lee (2015) studied thermal stresses in an isotropic material with an open penny-shaped crack filled with a heat-conducting medium.

Martynyak and Honchar (2005) investigated the temperature field and thermal stresses in an isotropic bi-material with an interface crack filled with a heat-conducting medium. The effect of the crack opening on the crack thermal resistance varying with mechanical and thermal loads was taken into account, and the bi-material components were supposed to have identical mechanical characteristics and different thermal characteristics. The similar problem was also considered (Martynyak and Serednytska, 2017) for the case of different mechanical and thermal characteristics of the bi-material components and the zero Dundurs parameter. Goldstein et al. (2014) studied the influence of thermal resistances of a heat-conducting filler and surface thin films on the partial closure of a crack.

In this paper, we will investigate thermo-elastic behavior of a bi-material that has a gas-filled interface crack and is subjected to a uniform tensile load and uniform heat flow, taking into account pressure and thermal resistance of the crack filler.

\section{Statement of the problem}

Consider the problem of linear thermoelasticity assuming plane strain conditions. The bi-material is a plane consisting of two components $D_{1}$ and $D_{2}$ (Fig. 1) which have different thermomechanical parameters: $\lambda_{1}, \lambda_{2}$ - thermal conductivities, $\alpha_{1}, \alpha_{2}$ - coefficients of linear thermal expansion, $\nu_{1}, \nu_{2}$ - Poisson's ratios, $G_{1}, G_{2}$ - shear moduli. We assume that the Dundurs parameter (Johnson, 1985)

$$
\beta=\frac{\left(1-2 \nu_{2}\right) G_{1}-\left(1-2 \nu_{1}\right) G_{2}}{2 G_{2}\left(1-\nu_{1}\right)+2 G_{1}\left(1-\nu_{2}\right)}
$$

of the bi-material is zero $(\beta=0)$. Then, the elastic parameters of the bi-material components are interconnected, and the relation $\left(1-2 \nu_{1}\right) G_{2}=\left(1-2 \nu_{2}\right) G_{1}$ takes place.

A crack is located at the bi-material interface. Its length equals $2 a$. A uniform tensile stress $p$ and a uniform steady heat flux $q$ are imposed distant from the crack $(y \rightarrow \pm \infty)$ in the direction normal to the interface. In addition, the bi-material components $D_{1}$ and $D_{2}$ are subjected to the stresses $S_{1}$ and $S_{2}(x \rightarrow \pm \infty)$, which are linearly distributed with respect to $y$. These stresses prevent global distortion and longitudinal deformation of the bi-material components due to tension $p$ and heat flux $q$. An increase in the tensile stress $p$ leads to the crack opening. The crack is supposed to be filled with a gas which exerts pressure $P_{g}$ on the crack surfaces and has thermal conductivity $\lambda_{g}$. As a mathematical model of the gas, the ideal gas model is chosen. The state of such a gas is described by the well-known ideal gas law. The influence of the filler on the heat transfer across the crack is modeled (Martynyak and Chumak, 2009) by thermal resistance which is directly proportional to the crack opening $h(x)$ and inversely proportional 


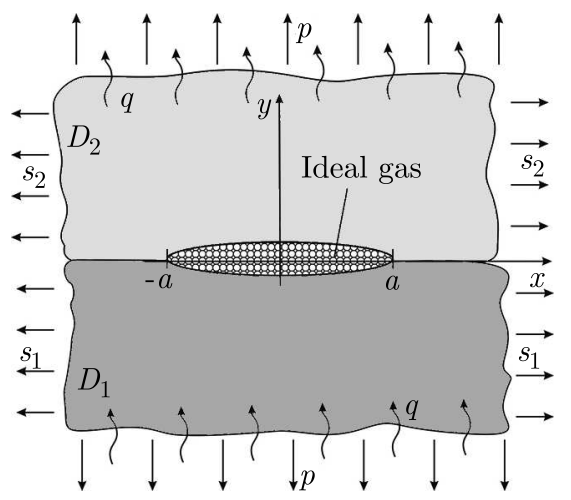

Fig. 1. Bi-material with an interface crack

to the filler thermal conductivity $\lambda_{g}$. At the interface outside the crack, the conditions of the perfect thermal and mechanical contact are assumed.

The thermal and mechanical boundary conditions are as follows:

— on the crack surfaces $(y=0,|x|<a)$

$$
\begin{array}{ll}
q_{y}^{+}=q_{y}^{-} & T^{-}-T^{+}=\frac{h(x)}{\lambda_{g}} q_{y}^{+} \\
\sigma_{y}^{+}=\sigma_{y}^{-} & \tau_{x y}^{+}=\tau_{x y}^{-} \quad \sigma_{y}^{-}=P_{g}
\end{array}
$$

- at the interface outside the crack $(y=0,|x| \geqslant a)$

$$
\begin{array}{lll}
T^{+}=T^{-} & q_{y}^{+}=q_{y}^{-} & \sigma_{y}^{+}=\sigma_{y}^{-} \\
\tau_{x y}^{+}=\tau_{x y}^{-} & u^{+}=u^{-} & v^{+}=v^{-}
\end{array}
$$

— at infinity

$$
\begin{array}{lll}
q_{y}^{\infty}=q & q_{x}^{\infty}=0 & \sigma_{y}^{\infty}=p \\
\tau_{x y}^{\infty}=0 & \sigma_{x_{1}}^{\infty}=S_{1} & \sigma_{x_{2}}^{\infty}=S_{2}
\end{array}
$$

Here and further on, $T$ is temperature; $q_{x}, q_{y}$ are the components of heat flow; $u, v-$ displacement components; $\sigma_{x}, \sigma_{y}$, and $\tau_{x y}$ are stress components; the superscripts "+" and "-_" denote the boundary values of a function on the $x$-axis in the upper and lower half-plane, respectively; $S_{n}(n=1,2)$ is the thermal distortivity of the material $D_{n}$ (Dundurs, 1974)

$$
S_{n}=\frac{2 G_{n} \eta_{n} q}{1-\nu_{n}} y+\frac{\nu_{n} p}{1-\nu_{n}} \quad \eta_{n}=\frac{\alpha_{n}\left(1+\nu_{n}\right)}{\lambda_{n}} \quad n=1,2
$$

\section{Solution to the problem}

Using the technique of solving thermoelastic problems for bi-materials with open cracks (Martynyak and Serednytska, 2017), we present the thermo-stressed state in the bi-material through a crack opening $h(x)=v^{+}-v^{-}$, a temperature jump $\gamma(x)=T^{-}-T^{+}$between the crack faces, and a tangential displacements jump $U(x)=u^{-}-u^{+}$. Satisfying conditions (2.1)-(2.3), we obtain a nonlinear system of singular integro-differential equations (SID equations) for functions $h(x)$, $\gamma(x)$ and $U(x)$ 


$$
\begin{array}{ll}
\frac{1}{\pi} \int_{-a}^{a} \frac{h^{\prime}(t)}{t-x} d t+\frac{\lambda \eta^{-}}{2} \gamma(x)=-\frac{1}{2 G^{*}}\left(p+P_{g}\right) & |x|<a \\
\lambda_{g} \frac{\gamma(x)}{h(x)}-\frac{\lambda}{2 \pi} \int_{-a}^{a} \frac{\gamma^{\prime}(t)}{t-x} d t=q \quad|x|<a & \\
\frac{1}{\pi} \int_{-a}^{a} \frac{U^{\prime}(t)}{t-x} d t-\frac{\lambda \eta^{+}}{2 \pi} \int_{-a}^{a} \frac{\gamma(t)}{t-x} d t=0 \quad|x|<a
\end{array}
$$

where $\lambda=2 \lambda_{1} \lambda_{2} /\left(\lambda_{1}+\lambda_{2}\right), G^{*}=G_{1}\left(1-\kappa_{2}\right) /\left(1-\kappa_{2}\right), \kappa_{n}=3-4 \nu_{n}, \eta^{-}=\eta_{2}-\eta_{1}, \eta^{+}=\eta_{1}+\eta_{2}$.

The desired crack opening $h(x)$, temperature jump $\gamma(x)$ and tangential displacements jump $U(x)$ must satisfy the following conditions at the ends of the crack

$$
h( \pm a)=0 \quad \gamma( \pm a)=0 \quad U( \pm a)=0
$$

Since the tangential displacements jump $U(x)$ does not enter Eqs. $(3.1)_{1}$ and $(3.1)_{2}$ of the system and depends only on the temperature jump $\gamma(x)$, we can solve the system of Eqs. $(3.1)_{1}$ and $(3.1)_{2}$ at first, and then find the solution to Eq. (3.1) 3 with the known $\gamma(x)$.

Taking into account third condition (3.2), we find a solution to singular integral equation $(3.1)_{3}$ that relates the function $U^{\prime}(x)$ with the temperature jump $\gamma(x)$

$$
U^{\prime}(x)=\frac{\lambda \eta^{+}}{2}\left(\gamma(x)-\frac{1}{\pi \sqrt{a^{2}-x^{2}}} \int_{-a}^{a} \gamma(x) d x\right)
$$

The gas pressure $P_{c}$ is determined from the ideal gas law

$$
P_{g} V_{g}=\frac{m_{g} R T_{g}}{\mu_{g}}
$$

Here, $m_{g}$ is gas mass, $V_{g}$ - gas volume per unit of the crack length $(l=1 \mathrm{~m})$ in the direction perpendicular to the plane of Fig. $1, \mu_{g}$ - gas molar mass, $T_{g}$ - absolute gas temperature, $R=8.3145 \mathrm{~J} /(\mathrm{K} \mathrm{mol})$ - the universal gas constant.

The gas volume can be expressed in terms of the crack opening as

$$
V_{g}=l \int_{-a}^{a} h(x) d x
$$

Taking into account that the temperature jump on the crack surfaces has a small value in comparison with the reference temperature, we assume that the absolute gas temperature is equal to the reference temperature

$$
T_{g}=T_{0}
$$

Substitution of expressions (3.5) and (3.6) into ideal gas law (3.4) yields the following relation between the gas pressure $P_{g}$ and the crack opening $h(x)$

$$
P_{g}=\frac{m_{g} R T_{0}}{\mu_{g}} l \int_{-a}^{a} h(x) d x
$$


Taking into account (3.7), the system of SID equations $(3.1)_{1},(3.1)_{2}$ can be rewritten as

$$
\begin{aligned}
& \frac{1}{\pi} \int_{-a}^{a} \frac{h^{\prime}(t)}{t-x} d t+\frac{\lambda \eta^{-}}{2} \gamma(x)+\frac{m_{g} R T_{0}}{2 G^{*} \mu_{g} l \int_{-a}^{a} h(x) d x}=-\frac{1}{2 G^{*}} p \quad|x|<a \\
& \lambda_{g} \frac{\gamma(x)}{h(x)}-\frac{\lambda}{2 \pi} \int_{-a}^{a} \frac{\gamma^{\prime}(t)}{t-x} d t=q \quad|x|<a
\end{aligned}
$$

The structure of system (3.8) indicates the fact that the solution of the considered problem essentially depends on the properties of the filler, characteristics of the materials and mechanical and thermal loads.

Let us consider the case of identical thermal distortivities of the materials $\left(\eta_{1}=\eta_{2}\right)$. Then $\eta^{-}=0$, and system (3.8) has the following form

$$
\begin{aligned}
& \frac{1}{\pi} \int_{-a}^{a} \frac{h^{\prime}(t)}{t-x} d t+\frac{m_{g} R T_{0}}{2 G^{*} \mu_{g} l \int_{-a}^{a} h(x) d x}=-\frac{1}{2 G^{*}} p \quad|x|<a \\
& \lambda_{g} \frac{\gamma(x)}{h(x)}-\frac{\lambda}{2 \pi} \int_{-a}^{a} \frac{\gamma^{\prime}(t)}{t-x} d t=q \quad|x|<a
\end{aligned}
$$

The system of SID equations (3.9) is partially separated. The function $\gamma(x)$ does not enter equation (3.9) ${ }_{1}$ and the crack opening $h(x)$ does not depend on the imposed heat flow. Nevertheless, the temperature jump $\gamma(x)$ depends on the crack opening $h(x)$ (see Eq. $(3.9)_{2}$ ). The temperature jump and temperature field in the bi-material are therefore dependent on the applied mechanical load and the gas pressure.

In the papers (Martynyak and Honchar, 2005) and (Martynyak and Serednytska, 2017), the investigation of the thermo-stressed state of a bi-material in the case of different thermal distortivities of bi-material components $\left(\eta_{1} \neq \eta_{2}, \eta^{-} \neq 0\right)$ was conducted. It took into account the heat conductivity of the crack filler but did not take into account its pressure. The functions $h(x)$ and $\gamma(x)$ were determined from the system of SID equations

$$
\begin{aligned}
& \frac{1}{\pi} \int_{-a}^{a} \frac{h^{\prime}(t)}{t-x} d t+\frac{\lambda \eta^{-}}{2} \gamma(x)=-\frac{1}{2 G^{*}} p \quad|x|<a \\
& \lambda_{g} \frac{\gamma(x)}{h(x)}-\frac{\lambda}{2 \pi} \int_{-a}^{a} \frac{\gamma^{\prime}(t)}{t-x} d t=q \quad|x|<a
\end{aligned}
$$

The system of SID equations (3.10) is non-linear and interconnected. The temperature and stresses in the bi-material are therefore interconnected too.

When thermal distortivities of the materials are different, changes of the coefficient of thermal conductivity $\lambda_{g}$ and heat flow density $q$ have different influence on the crack opening $h(x)$ for two opposite heat flow directions (Martynyak and Serednytska, 2017): heat flowing from the material with a smaller thermal distortivity into the material with a larger thermal distortivity $\left(q \eta^{-}>0\right)$ and, vice versa, heat flowing from the material with the larger thermal distortivity into the material with the smaller thermal distortivity $\left(q \eta^{-}<0\right)$. This effect is called as the heat flow directional effect or thermal rectification (Roberts and Walker, 2011).

In this paper, we investigate the heat flow directional effect taking into account the simultaneous influence of the thermal conductivity and pressure of the gas in the crack. The parameter $\eta^{-}$ 
is nonzero in system (3.8). To solve system (3.8), we will apply an analytico-numerical iteration procedure which is a generalization of the procedure developed in (Martynyak and Serednytska, 2017).

Mode I stress intensity factor $K_{\mathrm{I}}=\lim _{x \rightarrow a}\left\{\sqrt{2 \pi(x-a)} \sigma_{y}(x, 0)\right\}$ and Mode II stress intensity factor $K_{\mathrm{II}}=\lim _{x \rightarrow a}\left\{\sqrt{2 \pi(x-a)} \tau_{x y}(x, 0)\right\}$ in the vicinity of the right crack end are expressed through the temperature jump and the crack opening (Martynyak and Serednytska, 2017)

$$
K_{\mathrm{I}}=-2 G^{*} \lim _{x \rightarrow a}\left[\sqrt{2 \pi(a-x)} h^{\prime}(x)\right] \quad K_{\mathrm{II}}=-\frac{\lambda \eta^{+}}{G^{*}} \sqrt{\pi a} \int_{-a}^{a} \gamma(t) d t
$$

\section{Numerical calculations and analysis of results}

For numerical calculations, we introduce the following dimensionless values: coordinate $\bar{x}=x / a$, crack opening $\bar{h}=h / a$, temperature jump $\bar{\gamma}=\gamma \lambda \eta^{-}$, heat flux $\bar{q}=q a \eta^{-}$, tensile stress $\bar{p}=p / G^{*}$, gas pressure $\bar{P}_{g}=P_{g} / G^{*}$, gas thermal conductivity $\bar{\lambda}_{g}=\lambda_{g} / \lambda$, gas mass $\bar{m}_{g}=m_{g} R T_{0} /\left(G^{*} \mu_{g} a^{2} l\right)$, Mode I and Mode II stress intensity factors $\bar{K}_{\mathrm{I}}=K_{\mathrm{I}}\left(G^{*} \sqrt{a}\right)$ and $\bar{K}_{\mathrm{II}}=K_{\mathrm{II}} \eta^{-} /\left(G^{*} \eta^{+} \sqrt{a}\right)$.

System (3.9) in the dimensionless form is

$$
\begin{array}{lcc}
\frac{1}{\pi} \int_{-1}^{1} \frac{\bar{h}^{\prime}(t)}{t-\bar{x}} d t+\frac{1}{2} \bar{\gamma}(\bar{x})+\bar{m}_{g} /\left(2 \int_{-1}^{1} \bar{h}(\bar{x}) d \bar{x}\right)=-\frac{1}{2} \bar{p} \quad|\bar{x}|<1 & \bar{h}( \pm 1)=0 \\
\bar{\lambda}_{g} \frac{\bar{\gamma}(\bar{x})}{\bar{h}(\bar{x})}-\frac{\lambda}{2 \pi} \int_{-1}^{1} \frac{\bar{\gamma}^{\prime}(t)}{t-\bar{x}} d t=\bar{q} \quad|\bar{x}|<1 \quad \bar{\gamma}( \pm 1)=0 &
\end{array}
$$

The dimensionless heat flux is positive $(\bar{q}>0)$ when heat flows into the material with the larger thermal distortivity, and the heat flux is negative $(\bar{q}<0)$ when heat flows into the material with the smaller thermal distortivity.

The iteration procedure has different schemes for these two opposite directions of the heat flow.

For $\bar{q}>0$, the iterates of the desired functions are found from the following linear system of SID equations

$$
\begin{array}{ll}
\frac{1}{\pi} \int_{-1}^{1} \frac{\bar{h}^{(i)}(t)}{t-\bar{x}} d t+\frac{1}{2} \bar{\gamma}^{(i-1)}(\bar{x})+\bar{m}_{g} /\left(2 \int_{-1}^{1} \bar{h}^{(i-1)}(\bar{x}) d \bar{x}\right)=-\frac{1}{2} \bar{p} \quad|\bar{x}|<1 \\
\bar{\lambda}_{g} \frac{\bar{\gamma}^{(i)}(\bar{x})}{\bar{h}^{(i)}(\bar{x})}-\frac{\lambda}{2 \pi} \int_{-1}^{1} \frac{\bar{\gamma}^{\prime(i)}(t)}{t-\bar{x}} d t=\bar{q} & |x|<1 \\
\bar{h}^{(i)}( \pm 1)=0 \quad \bar{\gamma}^{(i)}( \pm 1)=0 &
\end{array}
$$

where $i=1,2, \ldots, \bar{\gamma}^{(0)}(\bar{x})=0, \bar{h}^{(0)}(\bar{x})=(\bar{p} / 2) \sqrt{1-\bar{x}^{2}}$. The initial iterate $\bar{h}^{(0)}(\bar{x})$ physically corresponds to the case when the heat flow is absent.

For $\bar{q}<0$, the iterates of the desired functions are found from the following linear system of SID equations 


$$
\begin{aligned}
& \frac{1}{\pi} \int_{-1}^{1} \frac{\bar{h}^{(i)}(t)}{t-\bar{x}} d t+\left(\frac{\bar{q}}{2 \bar{\lambda}_{g}}+\frac{1}{4 \pi \bar{\lambda}_{g}} \int_{-1}^{1} \frac{\bar{\gamma}^{(i-1)}(t)}{t-\bar{x}} d t\right) \bar{h}^{(i)}(\bar{x})+\bar{m}_{g} /\left(2 \int_{-1}^{1} \bar{h}^{(i-1)}(\bar{x}) d \bar{x}\right)=-\frac{1}{2} \bar{p} \\
& \bar{\lambda}_{g} \frac{\bar{\gamma}^{(i)}(\bar{x})}{\bar{h}^{(i)}(\bar{x})}-\frac{1}{2 \pi} \int_{-1}^{1} \frac{\bar{\gamma}^{\prime(i)}(t)}{t-\bar{x}} d t=\bar{q} \quad|\bar{x}|<1 \\
& \bar{h}^{(i)}( \pm 1)=0 \quad \bar{\gamma}^{(i)}( \pm 1)=0
\end{aligned}
$$

where $i=1,2, \ldots, \bar{\gamma}^{(0)}(\bar{x})=0, \bar{h}^{(0)}(\bar{x})=\left[\bar{p} \bar{\lambda}_{g} /\left(2 \bar{\lambda}_{g}-\bar{q}\right)\right] \sqrt{1-\bar{x}^{2}}$. In this case, the initial iterate $\bar{h}^{(0)}(\bar{x})$ takes into account thermal strains in the bi-material.

Systems (4.2) and (4.3) are solved using the collocation method (Gabdulkhaev, 1994).

The iteration process is finished when

$$
\left|\frac{h^{(i)}(0)-h^{(i-1)}(0)}{h^{(i-1)}(0)}\right| \leqslant 10^{-5} \quad\left|\frac{\gamma^{(i)}(0)-\gamma^{(i-1)}(0)}{\gamma^{(i-1)}(0)}\right| \leqslant 10^{-5}
$$

The crack opening $\bar{h}(\bar{x})$, temperature jump $\bar{\gamma}(\bar{x})$, gas pressure $\bar{P}_{g}$ and stress intensity factors $\bar{K}_{\mathrm{I}}$ and $\bar{K}_{\mathrm{II}}$ are analyzed for a given set of the input parameters $\bar{p}, \bar{q}, \bar{\lambda}_{g}, \bar{m}_{g}$, and $\bar{T}_{0}$. The results of numerical calculations are shown for $\bar{p}=0.01$ and the two opposite directions of heat flow. The solid curves correspond to the case of heat flowing into the material with the larger thermal distortivity $(\bar{q}>0)$, while dashed curves correspond to the case of heat flowing into the material with the smaller thermal distortivity $(\bar{q}<0)$.

Figure 2a illustrates the crack opening $\bar{h}(\bar{x})$ for different values of the gas mass $\bar{m}_{g}, \bar{\lambda}_{g}=0.01$ and $\bar{q}= \pm 0.01$. Curves 1 and 4 correspond to the case when the gas exerts no pressure on the crack surfaces. The crack opening increases with the increasing mass pf the gas for the two opposite heat flow directions. For fixed values of the input parameters, the crack opening in the case of heat flow into the material with the larger thermal distortivity is observed to be greater than the crack opening in the case of the opposite heat flow direction.

A noteworthy feature of the results shown in Fig. 2a is that the crack opening changes upon reversal of the direction in which heat is flowing. Consequently, the heat flow directional effect takes place.

Figure $2 \mathrm{~b}$ shows an increase of the absolute value of the temperature jump $|\bar{\gamma}(\bar{x})|$ with an increase of the gas mass $\bar{m}_{g}$ for both heat flow directions. The distribution of the temperature jump is qualitatively similar to the crack opening distribution.
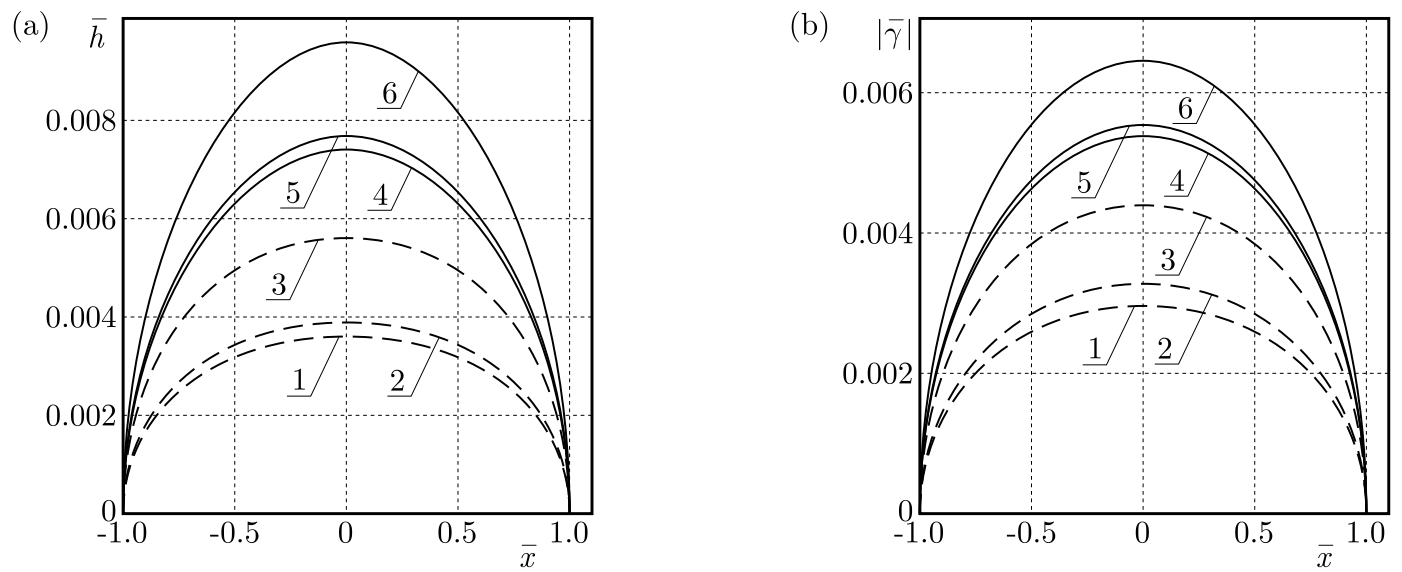

Fig. 2. (a) The crack opening $\bar{h}(\bar{x})$ and (b) the absolute value of the temperature jump $|\bar{\gamma}(\bar{x})|$ for $\bar{\lambda}_{g}=0.01$ and various values of $\bar{m}_{g}: 1,4-\bar{m}_{g}=0 ; 2,5-\bar{m}_{g}=0.00001 ; 3,6-\bar{m}_{g}=0.0001$ 
The dependences of the gas pressure $\bar{P}_{g}$ on the gas mass $\bar{m}_{g}$, the gas thermal conductivity $\bar{\lambda}_{g}$ and the absolute value of the heat flux $|\bar{q}|$ for both heat flow directions are given in Figs. 3a and $3 \mathrm{~b}$. The gas pressure increases with the increasing mass of the gas and is greater in the case of heat flowing into the less distortive material. An increase in the gas thermal conductivity $\bar{\lambda}_{g}$ or the absolute value of the heat flux $|\bar{q}|$ leads to a decrease in the gas pressure $\bar{P}_{g}$ when $\bar{q}<0$ and an increase in $\bar{P}_{g}$ when $\bar{q}>0$. The difference between gas pressure values increases with decreasing $\bar{\lambda}_{g}$ and increasing $|\bar{q}|$ for both heat flow directions.
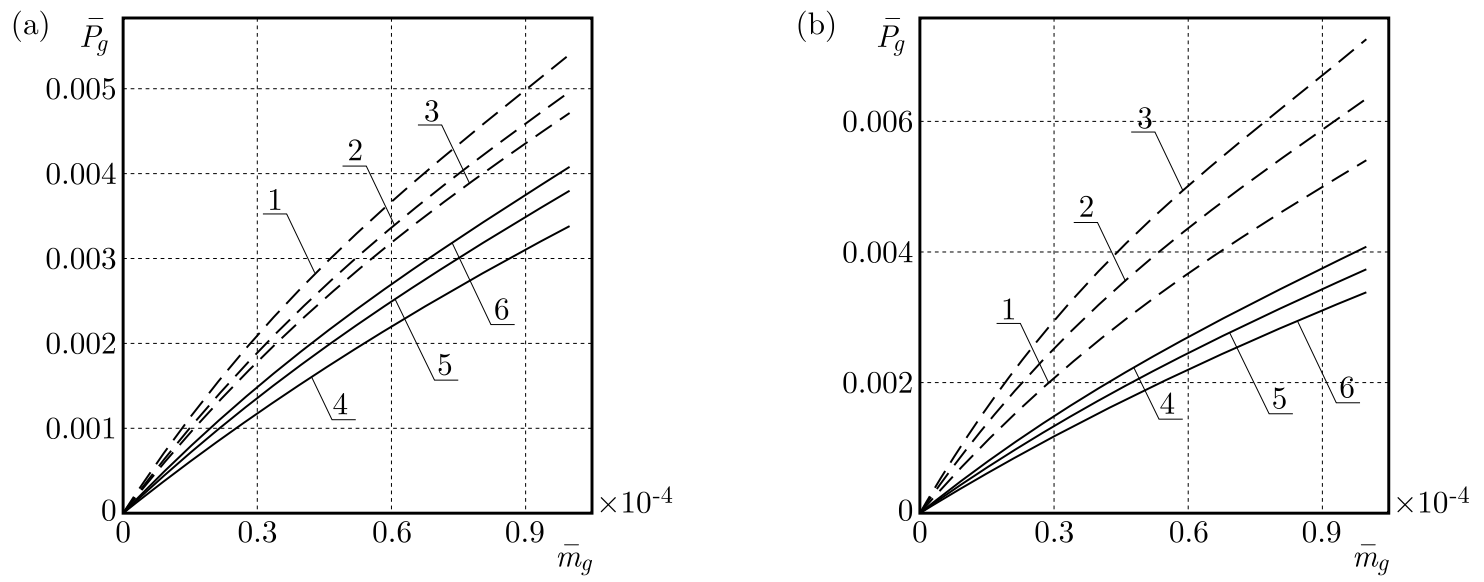

Fig. 3. The gas pressure $\bar{P}_{g}$ vs. gas mass $\bar{m}_{g}$ : (a) for $|\bar{q}|=0.01$ and various values of $\bar{\lambda}_{g}: 1,4-\bar{\lambda}_{g}=0.01$; $2,5-\bar{\lambda}_{g}=0.02 ; 3,6-\bar{\lambda}_{g}=0.04$ and (b) for $\bar{\lambda}_{g}=0.01$ and various values of $|\bar{q}|: 1,4-|\bar{q}|=0.01$; $2,5-|\bar{q}|=0.02 ; 3,6-|\bar{q}|=0.03$

The dependences of Mode I stress intensity factor $\bar{K}_{\mathrm{I}}$ and the absolute value of Mode II stress intensity factor $\left|\bar{K}_{\mathrm{II}}\right|$ on the gas mass $\bar{m}_{g}$ are shown in Figs. $4 \mathrm{a}$ and $4 \mathrm{~b} . \bar{K}_{\mathrm{I}}$ and $\left|\bar{K}_{\mathrm{II}}\right|$ increase with the increasing mass of the gas $\bar{m}_{g}$ and are greater in the case of the heat flow to the material with the larger thermal distortivity. $\bar{K}_{\mathrm{I}}$ increases with the increasing heat flux $|\bar{q}|$ when $\bar{q}>0$. For the opposite heat flow direction, $\bar{K}_{\mathrm{I}}$ decreases with increasing $|\bar{q}| \cdot\left|\bar{K}_{\mathrm{II}}\right|$ increases with increasing $|\bar{q}|$ for both heat flow directions (Fig. 4b). The difference between the values of the stress intensity factors increases with an increase in the heat flux for both heat flow directions.

(a)

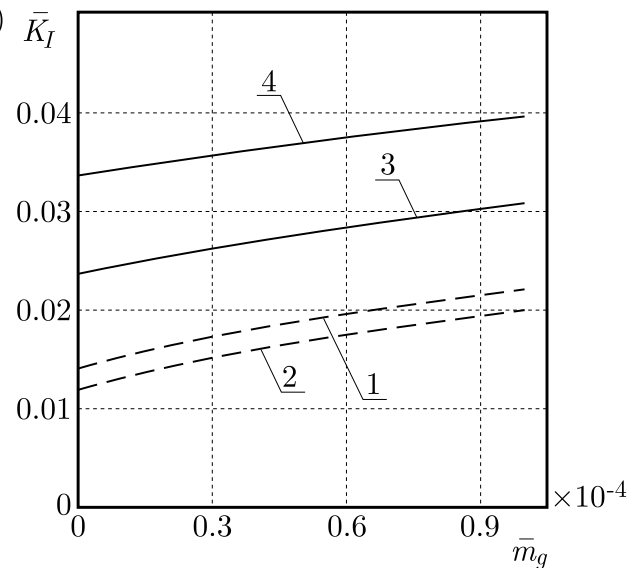

(b)

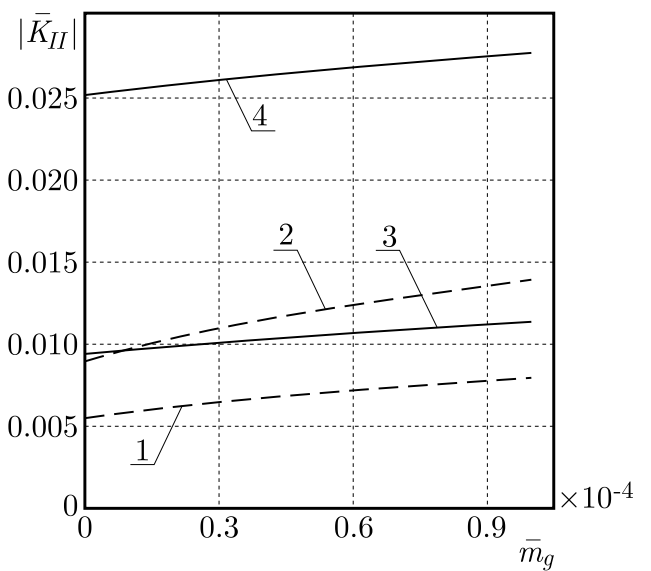

Fig. 4. (a) Mode I stress intensity factor $\bar{K}_{\mathrm{I}}$ and (b) the absolute value of Mode II stress intensity factor $\left|\bar{K}_{\mathrm{II}}\right|$ vs. gas mass $\bar{m}_{g}$ for $\bar{\lambda}_{g}=0.01$ and various values of $|\bar{q}|: 1,3-|\bar{q}|=0.01 ; 2,4-|\bar{q}|=0.02$

The stress intensity factors $\bar{K}_{\mathrm{I}}$ and $\left|\bar{K}_{\mathrm{II}}\right|$ versus the gas thermal conductivity $\bar{\lambda}_{g}$ are shown in Figs. $5 \mathrm{a}$ and $5 \mathrm{~b}$ for various values of the gas mass $\bar{m}_{g}$. With the increasing $\bar{\lambda}_{g}, \bar{K}_{\mathrm{I}}$ increases 
when $\bar{q}<0$ and decreases when $\bar{q}>0$. $\left|\bar{K}_{\text {II }}\right|$ decreases with increasing $\bar{\lambda}_{g}$ for both heat flow directions. The difference in the values of the stress intensity factors increases with a decrease in the gas thermal conductivity, but does almost not change with a change of the gas mass. It can also be seen that the stress intensity factors are less when the gas pressure is zero (curves 1 and 4 in Figs. 5a and 5b).
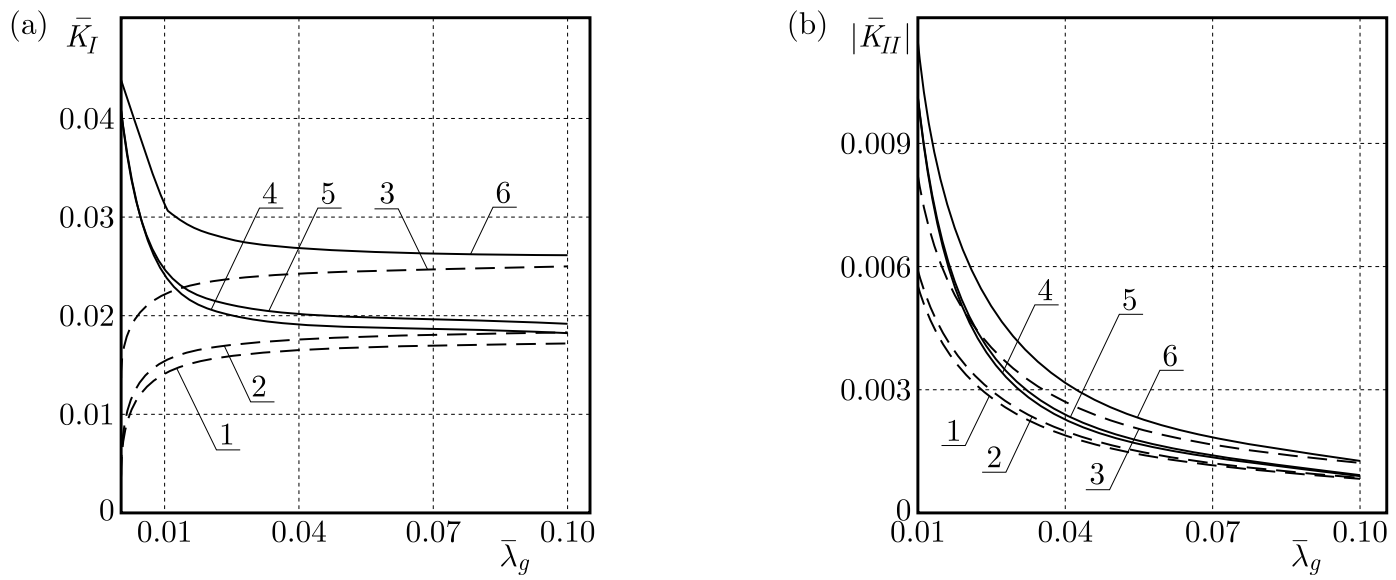

Fig. 5. (a) Mode I stress intensity factor $\bar{K}_{\mathrm{I}}$ and (b) the absolute value of Mode II stress intensity factor $\left|\bar{K}_{\mathrm{II}}\right|$ vs. gas thermal conductivity $\bar{\lambda}_{g}$ for $|\bar{q}|=0.01$ and various values of $\bar{m}_{g}: 1,4-\bar{m}_{g}=0$; $2,5-\bar{m}_{g}=0.00001 ; 3,6-\bar{m}_{g}=0.0001$

\section{Conclusions}

The thermoelastic problem for a bi-material with an interface crack filled with an ideal gas has been considered. Both thermal (thermal conductivity) and mechanical (pressure) influences of the gaseous crack filler have been taken into account. The heat transfer across the crack has been simulated by the thermal resistance which is directly proportional to the crack opening and inversely proportional to the gas thermal conductivity. The gas pressure has been determined using the ideal gas law equation. The problem under consideration has been reduced to a nonlinear system of singular integro-differential equations, and the method of successive approximations has been applied for its solving. The dependences of the crack opening, temperature jump, gas pressure as well as Mode I and Mode II stress intensity factors on the gas mass, gas thermal conductivity and heat flux have been analyzed.

All the parameters analyzed change upon reversal of the direction in which heat is flowing. Consequently, the bi-material with the heat-conducting crack exhibits the heat flow directional effect.

It has been revealed that the gas pressure increase contributes to the crack opening. An increase in the gas thermal conductivity leads to a decrease in the gas pressure when heat flows into a less distortive material and an increase in the gas pressure when heat flows into the material with a larger thermal distortivity. For the two opposite heat flow directions, Mode II stress intensity factor increases with the increasing absolute value of the heat flux or decreasing gas thermal conductivity. On the other hand, an increase in the heat flux absolute value or a decrease in the gas thermal conductivity leads to an increase in Mode I stress intensity factor when heat flows into the material with the larger distortivity and its decrease in the case of the opposite heat flow direction. For both heat flow directions, the stress intensity factors increase when the gas mass increases and are smaller when the gas pressure is zero. 


\section{References}

1. Alekseev A.D., Revva V.N., Volodarskiy Ye.V., Yurchenko V.M., 1992, Discharge of gas from solid solution into the volume and on the surface of pores and cracks (in Russian), Physics and High Pressure Technology, 2, 4, 83-92

2. Andreikiv O.Ye., Hembara O.V., 2008, Fracture Mechanics and Durability of Metal Materials in Hydrogenous Media (in Ukrainian), Naukova Dumka, Kyiv

3. Balueva A.V., Goldstein R.V., 1995, Method for calculating the kinetics of cracks in a medium with volume gas emission (in Russian), Izvestiya RAN. Mekhanika Tverdogo Tela, 3, 149-159

4. Chumak K., Martynyak R., 2012, Thermal rectification between two thermoelastic solids with a periodic array of rough zones at the interface, International Journal of Heat and Mass Transfer, 55, 21-22, 5603-5608

5. Dundurs J., 1974, Distortion of a body caused by free thermal expansion, Mechanics Research Communications, 1, 3, 121-124

6. Evtushenko A.A., Sulim G.T., 1981, Stress concentration near a cavity filled with a liquid, Materials Science, 16, 6, 546-549

7. Feraille-Fresnet A., Bui H.-D., Ehrlacher A., 2003, Hydrostatic interaction of a wetting fluid and a circular crack in an elastic material, Mechanics of Materials, 35, 3-6, 581-586

8. Gabdulkhaev B.G., 1994, The Direct Methods for Solving the Singular Integral Equations of the First Kind (in Russian), Izd. Kazanskogo Universiteta, Kazan

9. Goldstein R.V., Kit H.S., Martynyak R.M., Serednytska Kh.I., 2014, Effect of partial closure of an interface crack with heat-conducting filler and surface films in the case of thermal loading of a bimaterial, Journal of Mathematical Sciences, 198, 1, 75-86

10. Johnson K.L., 1985, Contact Mechanics, Cambridge University Press, Cambridge

11. KacZyŃski A., Monastyrskyy B., 2004, On the problem of some interface defect filled with a compressible fluid in a periodic stratified medium, Journal of Theoretical and Applied Mechanics, 42, $1,41-57$

12. Li X.-F., LEE K.Y., 2015, Effect of heat conduction of penny-shaped crack interior on thermal stress intensity factors, International Journal of Heat and Mass Transfer, 91, 127-134

13. Martynyak R.M., Chumak K.A., 2009, Thermoelastic delamination of bodies in the presence of a heat-conducting filler of the intercontact gap, Materials Science, 45, 4, 513-522

14. Martynyak R.M., Honchar Kh.I., 2005, Thermoelastic deformation of a bimaterial with an interface defect filled with heat-conducting substance (in Russian), Teoreticheskaya i Prikladnaya Mechanika, 41, 58-62

15. Martynyak R.M., Serednytska Kh.I., 2017, Contact Problems of Thermoelasticity for Interface Cracks in Bimaterials, Publishing House "Rastr-7", Lviv, Ukraine

16. Matczyński M., Martynyak R., Kryshtafovych A., 1999, Contact problem of a crack filled with heat-conducting gas, Thermal Stresses'99: Proceedings of the 3rd International Symposium on Thermal Stresses, 127-130

17. Monastyrskyy B., KaczyŃski A., 2007, The elasticity problem for a stratified semi-infinite medium containing a penny-shaped crack filled with a gas, Acta Mechanica et Automatica, 1, 1, $63-66$

18. Podstrigach Y.S., Kit G.S., 1967, Determination of temperature fields and stresses in the vicinity of heat-conducting cracks (in Russian), Termicheskie Napryazheniya v Elementakh Konstruktsiy, 7, 194-201

19. Podstrigach Y.S., 1963, A temperature field in the system of solids conjugated by a thin interstitial layer, Journal of Engineering Physics, 6, 10, 129-136 
20. Shvets R.M., Martynyak R.M., 1985, Integral equations of the contact problem of thermoelasticity for rough bodies (in Ukrainian), Dopovidi AN URSR. Seriya A, 11, 37-40

21. Roberts N.A., WAlker D.G., 2011, A review of thermal rectification observations and models in solid materials, International Journal of Thermal Sciences, 50, 648-662

22. Zhong X.-C., Lee K.Y., 2012, A thermal-medium crack model, Mechanics of Materials, 51, 110-117

23. Zhong X.-C., Wu B., 2012, Thermoelastic analysis for an opening crack in an orthotropic material, International Journal of Fracture, 173, 1, 49-55

Manuscript received August 22, 2018; accepted for print October 24, 2018 